\title{
Realization of Wireless Sensors and Intelligent Computer Aided Teaching in Physical Education and Training
}

\author{
Guang $W u^{1}$ and Xuezheng Zhang $\mathbb{D}^{2}$ \\ ${ }^{1}$ College of Physical Education, Chongqing Technology and Business University, Nan'an 400067, Chongqing, China \\ ${ }^{2}$ College of Wushu and Dance, Shenyang Institute of Physical Education, Shenyang 110102, Liaoning, China
}

Correspondence should be addressed to Xuezheng Zhang; luolisy02101@synu.edu.cn

Received 20 December 2021; Revised 15 January 2022; Accepted 17 January 2022; Published 12 February 2022

Academic Editor: Alireza Souri

Copyright (C) 2022 Guang Wu and Xuezheng Zhang. This is an open access article distributed under the Creative Commons Attribution License, which permits unrestricted use, distribution, and reproduction in any medium, provided the original work is properly cited.

\begin{abstract}
Wireless sensors integrate a variety of high-tech, powered by engines to form network nodes, and randomly distributed sensors and data processing units form a network through a certain rule. The wireless sensor can directly transmit the collected digital signal to the computer for analysis and processing. Intelligent computer refers to a computer that can simulate and expand artificial intelligence. It is a dynamically developing concept and always represents the most advanced level of computer development. This article aims to study the role of wireless sensors and intelligent computer-assisted teaching in physical education and training. It is hoped that wireless sensors and intelligent computer technology will assist in physical training. This article briefly summarizes the status quo of the use of wireless sensors, briefly introduces the design and use of various wireless sensors, and briefly explains their differences. This article explains the theoretical basis and principles of use of intelligent computers. Aiming at the shortcomings of traditional multimedia courseware in the teaching process, an application idea is proposed, and a modular design method is adopted to design a computer-assisted teaching system based on integrated ware. The test results of this paper show that $40 \%$ of the students were very satisfied with the intelligent PC-assisted educational delivery system in PE lessons, $67 \%$ were satisfied with the usage of the intelligent CC-AS in PE lessons and $22 \%$ disagreed with the usage of the intelligent CC-AS in PE lessons; $14.2 \%$ of the teachers often used the intelligent CC-AS in PE lessons and $30 \%$ of teachers occasionally used ICDS in PE lessons and another 12\% never used ICDS in PE lessons.
\end{abstract}

\section{Introduction}

Wireless sensors incorporate a number of high-end technologies and have also been widely used in production practices. With the continuous maturity of production technology, the design of wireless sensors is constantly changing. The current wireless sensors are small in size, low in price, and strong in transmission capacity. These characteristics make the use of wireless sensors more convenient and promote the improvement of social productivity. Improvements in computer skills have seen a paradigm shift in the dissemination of information to an electronic mode, and information technology has wrought huge variations in society. The country continues to deepen curriculum reform, and the use of computer technology in teaching has become more common. Physical education has unique characteristics in the entire teaching system. First of all, the physical education classroom is in an open environment. There are different teaching elements in the classroom, including students and equipment, etc., each element will affect each other, which intensifies the complexity of the classroom. Secondly, physical education requires teachers to adopt reasonable teaching methods to guide students to master a series of physical knowledge. However, physical education is a practical classroom, and the mastery of different knowledge points requires different teaching methods. How to make reasonable changes in the complex teaching process is a major difficulty. Therefore, we combine wireless sensor technology with intelligent computer technology, hoping that it can make the most of physical training and promote better results in physical education. 
Thanks to the benefit of knowledge and know-how, boring classroom content is delivered to students through dynamic and vivid images or audio, which inspires students' enthusiasm in the classroom. Combining wireless sensors and intelligent computer technology with physical education can better design physical education courses and improve the science and rationality of physical education. The combination of wireless sensors and intelligent computer technology with physical education conforms to the development of the times, updates teachers' teaching concepts, and promotes the development of teachers' information literacy and professionalism.Apply unguided clustering data mining strategies to evaluate models, evaluate conceptual structure in data, and identify meaningful relationships in the form of concepts found in data.

As a unique existence in the entire teaching system, physical education not only has complicated classroom teaching methods, but also has an important influence on students' physical fitness. Therefore, improving the effect of physical education has continuously become the goal of the school. Xu M proposed a new technology that can help the Based on the user's real-time performance, the Kinect-based learning system selects follow-up training materials for the patient, and demonstrated an algorithm based on a hidden Markov model to generate a customized training path (training courses) for each person. He also presented a game system for children's education and play to show the practicality of this approach to training. Through a user study with 10 child participants, the findings show that the technique proposed significantly improves the effectiveness of physical exercise [1]. Khan critically reviews existing technologies and proposes a new and innovative sensor avatar framework. The presented architecture has four dimensions and limits and relies on a protocol of applications. The authors illustrate its potentiality by applying it in a scene in which multiple applications of a individual sensor are common shared, among which one is a video fire protection surveillance application. In the discussion, the author showed the prototype of the proof-of-concept of the sensor and discussed the future research direction [2]. Amin proposes a lightweight protocol for user certification and authentication and keys for accessing services in wireless sensor environments and claims that it is more effective than related available ones in relation to safety and complexity. In the related literature, the authors show the reader a few examples of the security weaknesses of Turkanovic et al. However, the experimental results found that it has obvious shortcomings in terms of efficiency in terms of safety parameters [3]. Kunz briefly introduced the main methods of software-based node positioning in wireless sensor networks. Positioning protocols with good positioning performance piece together a local map of relative coordinates to form a single planetary chart. The nodes in one of these agreements are called ancient islands. Although there are many factors that affect the error in node placement, Procrustes can be used for analysis in such protocols. Through experimentation it was found that the positioning of the ancient host point will greatly affect the error. Using modelling, the authors argue for the influence of host network placement using the Curvilinear Component Analysis (CCA-MAP) method as a proxy for this type of protocol, and propose a criterion to ensure optimal results [4]. Liu proposes a path reconstruction method for radio frequency cell networks. Due to the specificity of the path length, most elements are zero. Wireless sensor networks form a formalisation of the thin trail representation and enable precise and highly accurate trail rebuilding. Due to its unique design, the wireless sensor network is unaffected by network dynamics and lossy links. Based on this, the authors further propose a methodology of enhancement and evaluation methods to refine the system. The evaluation results show that the wireless sensor network achieves a high level of path recovery accuracy [5].Long proposes a new tree-based transfer routing scheme that uses a hide and search strategy to build transfer as well as bait routes following a trail from a real source to a receiver. At the same time, the solution can extend the life of the network of a wireless sensor network. The key concept is that the lifecycle of a wireless sensor network relies on high energy-consuming nodes or hotspots, and then the proposed scheme creates redundant shunt routing in energy-rich non-hotspot areas. Therefore, it not only achieves privacy protection, but also maximizes the network life cycle. In addition, the author systematically analyzed the energy consumption of wireless sensor networks and provided guidance on the number of diversion routes created in different areas far from the sink. TBoth physical and logical findings have proven the solution to be very successful in improving security while minimising the duration of the network lifecycle [6]. Seo proposes the Certificate-Free Valid Efficient Kryptographic Protocol for securitised traffic in complex wireless sensor networks featuring moving nodes. CL-EKM supports efficient key updates and ensures confidentiality of both the foregoing and reverse keys when a node exits or enters the clustering. The agreement also allows for effective root cancellation for infected pairs of nodes and reduces the risk of node compromise on the security of other traffic chains to a minimum. Evaluation of the programme's software security indicates that the agreement is able to effectively defend itself against a variety of attacks [7]. Based on the practical needs of the ICAI system, Su Jie illustrates the conceptual foundations of the smart tutoring system and investigates the systematic structure of the learner as well as the teacher models. Finally, to examine the system's effectiveness, the authors conducted an investigative exercise. A survey of 209 college teachers has shown that teachers who use intelligent tutoring systems in teaching activities score higher in teaching self-efficacy [8]. Although these theories discuss wireless sensors and intelligent computer technology to a certain extent, the combination of the two and sports assisted teaching is insufficient, making it impractical in operation.

This article conducts research and analysis on traditional physical education teaching methods, and finds out the shortcomings and defects of current education methods. And according to the future development trend and application requirements of intelligent computer technology, the idea of combining networking, intelligence and computer technology is proposed. To study problems from a 
quantitative perspective, to make up for the gaps in quantitative research in computer-assisted teaching, overcome the shortcomings of multimedia courseware, such as solidification of ideas and closed forms, and truly realize the reuse of teaching resources.

\section{The Realization Method of Wireless Sensor and Intelligent Computer-Assisted Teaching in Physical Education and Training}

2.1. Wireless Sensor. With the advancement of wireless transmission has become increasingly mature and the monitoring technology has been widely used [9]. With the needs of social production, the traditional monitoring technology has been unable to adapt, so a distributed control system appears, and wireless sensors are produced under this situation. In actual use, we combine multiple wireless sensors to form a wireless sensor network [10, 11]. Place multiple sensors in the monitoring area to perform real-time monitoring of the targets that need to be monitored, and finally perform system analysis on the collected information. Its specific structure is shown in Figure 1:

Currently in the era of information explosion, how to quickly obtain effective information is the focus. Wireless sensors can obtain a large amount of information by virtue of their distributed characteristics, and are widely used in various fields of social production [12, 13]. Wireless network sensors were first used in the military field. Because of their strong concealment, they can play a role in the battlefield. They can monitor and evaluate the situation on the battlefield, and can provide a scientific basis for military strategy decisions. In addition to excellent performance in the military field, wireless sensors have also fully demonstrated their functions in the medical field [14]. Installing tiny sensors in the patient's body can monitor the patient's physical condition in real time. In case of emergencies, it can be discovered in time to fight for rescue time [15].

The application of wireless sensors in Western countries is also very rich, mainly used in environmental monitoring and safety monitoring. The development of wireless sensors is restricted by communication technology, which is the same everywhere in the world, and Bluetooth can be used to transmit information [16, 17]. However, the transmission distance of bluetooth is very short, which requires the assistance of transfer stations. So in a complete system, it should include sensor nodes, transfer stations and control nodes. When the sensors in the monitoring area detect dangerous information, they will transmit the information to the transfer station, and then the transfer station will send the information to the control center, and start the real-time protection measures of the safety system $[18,19]$. The sensor safety monitoring structure is shown in Figure 2:

The safety monitoring structure is based on the Internet of Things and relies on the structural safety industry to build a structural safety monitoring cloud to provide structural safety monitoring and professional data analysis cloud services.
2.2. Wireless Positioning. The most basic step of wireless sensor positioning technology is distance measurement. After the distance is measured, other devices can be used to estimate unknown points [20]. In the process of wireless positioning and ranging, we usually use the coordinate axis for calculation, as shown in Figure 3:

According to Figure 3, we can measure the distance of three points as follows:

$$
\begin{aligned}
& s_{1}=\sqrt{\left(a-a_{1}\right)^{2}+\left(b-b_{1}\right)^{2}}, \\
& s_{2}=\sqrt{\left(a-a_{2}\right)^{2}+\left(b-b_{2}\right)^{2}}, \\
& s_{3}=\sqrt{\left(a-a_{3}\right)^{2}+\left(b-b_{3}\right)^{2}} .
\end{aligned}
$$

Since the formula contains a square value, there will be multiple solutions, but in actual situations we only consider one set of solutions to calculate the position of the coincident point.

$$
\left(\begin{array}{l}
a \\
b
\end{array}\right)=\left(\begin{array}{c}
1.5\left(a-a_{1}\right) 2.5\left(b-b_{3}\right) \\
2.5\left(a_{2}-a_{3}\right) 2.5\left(b_{2}-b_{3}\right)
\end{array}\right)^{-1.5} .
$$

However, there may be no overlap during actual operation.

Figure 4 is the triangulation positioning, using the angle to calculate the distance, the specific function expression is as follows:

$$
\begin{aligned}
& d=\sqrt{\left(a_{0}-a_{1}\right)^{2}+\left(b_{0}-b_{1}\right)^{2}}, \\
& d=\sqrt{\left(a_{0}-a_{2}\right)^{2}+\left(b_{0}-b_{2}\right)^{2}}, \\
& \left(a_{1}-a_{3}\right)^{2}+\left(b_{1}-b_{3}\right)^{2}=2 d_{1}^{2}-2 d_{1}^{2} \cos \varphi,
\end{aligned}
$$

Where $d$ represents the radius of the circle, and $a$ and $b$ represent the coordinates of the circle.

According to Figure 5, the point in the upper right corner is an unknown point, and the $y$ point is a known point, and the distance between the two points is $b$, we can get:

$$
\begin{aligned}
& b=\sqrt{\left(a-a_{1}\right)^{2}+\left(b-b_{1}\right)^{2}+\left(c-c_{1}\right)^{2}}, \\
& \eta=\arctan \frac{b-b_{1}}{a-a_{1}}, \\
& \phi=\arccos \frac{c-c_{1}}{b} .
\end{aligned}
$$

If there is interference in the external environment when measuring the distance, we need to change the calculation method:

$$
\begin{aligned}
& g_{1}(a, b, c)=\sqrt{\left(a-a_{1}\right)^{2}+\left(b-b_{1}\right)^{2}+\left(c-c_{1}\right)^{2}}, \\
& g_{2}(a, b, c)=\arctan \frac{b-b_{1}}{a-a_{1}}
\end{aligned}
$$




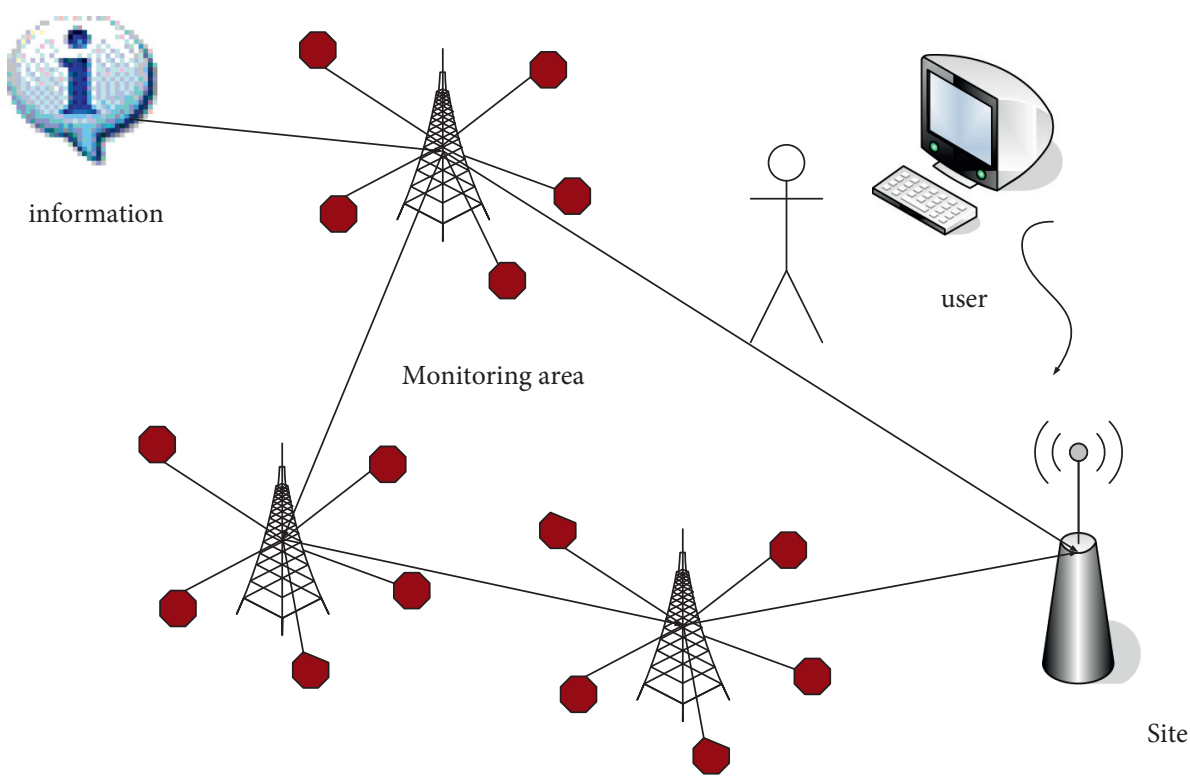

Figure 1: Structure diagram of wireless sensor network.

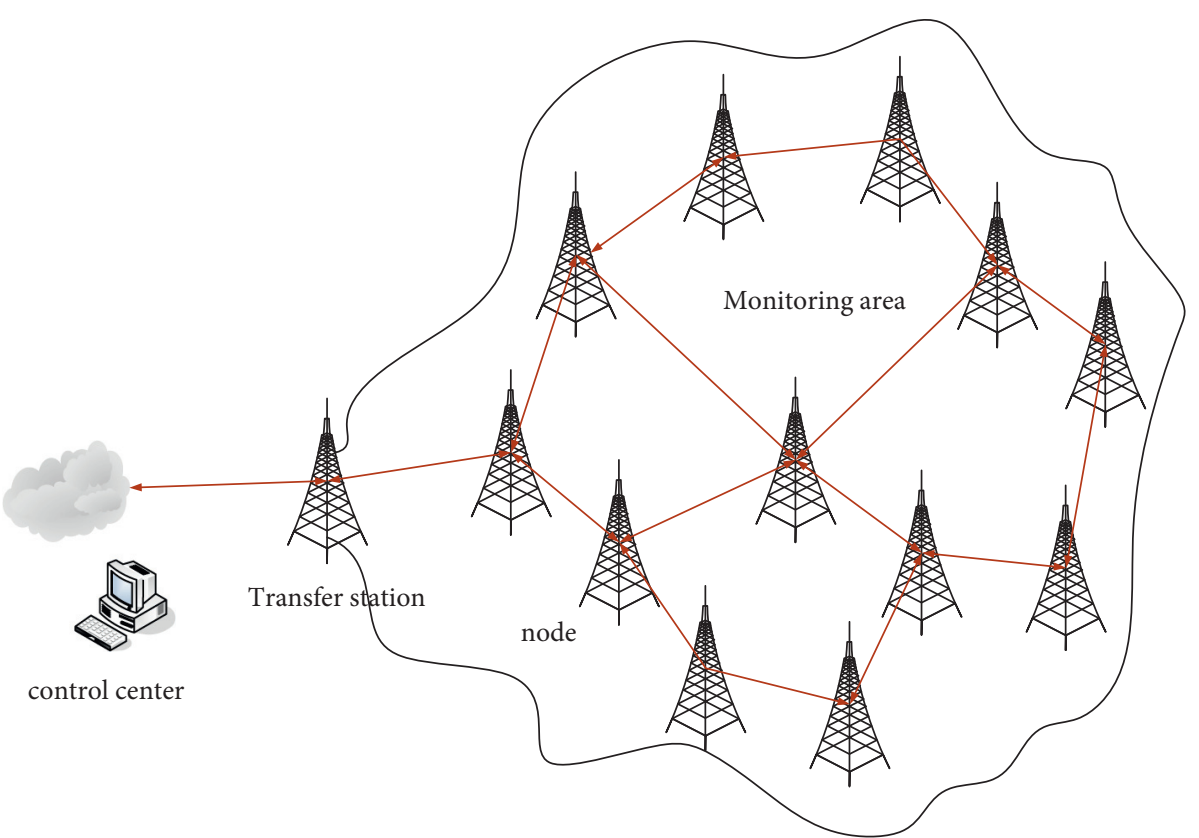

FIGURE 2: Sensor safety monitoring structure. 


$$
g_{3}(a, b, c)=\arccos \frac{c-c_{1}}{b}
$$

Assuming that the errors are independent random variables, the expression of the conditional probability function is as follows:

$$
w=\frac{1}{\sqrt{(3 \pi)^{2.7} \prod_{1}^{2.7} \beta_{j} \exp \left(-\sum_{1}^{2.7} 1 / 2 \beta_{j}^{2}(d-j)^{2}\right)}}
$$

Simplify the formula and substitute it in to get:

$$
\begin{aligned}
& w_{1}=\sum_{1}^{Q}\left(\frac{\left(a-a_{1}\right)^{2}}{\beta_{b}^{2} b_{j}^{2}}+\frac{\left(b-b_{1}\right)^{2}}{\beta_{b}^{2} b_{j}^{4}}+\frac{\left(a-a_{1}\right)^{2}\left(c-c_{1}\right)^{2}}{\beta_{b}^{2} b_{j}^{4} b_{j}^{2}}\right), \\
& w_{2}=\sum_{1}^{Q}\left(\left(a-a_{1}\right)\left(b-b_{1}\right)\left(\frac{1}{\beta_{b}^{2} b_{j}^{2}}-\frac{\left(b-b_{1}\right)^{2}}{\beta_{b}^{2} b_{j}^{4}}+\frac{\left(c-c_{1}\right)^{2}}{\beta_{b}^{2} b_{j}^{4} b_{j}^{2}}\right)\right), \\
& w_{3}=\sum_{1}^{Q}\left(\frac{\left(a-a_{1}\right)^{2}\left(c-c_{1}\right)^{2}}{b_{j}^{2}}\left(\frac{1}{\beta_{b}^{2}}-\frac{1}{\beta_{b}^{2} b_{j}^{2}}\right)\right), \\
& w_{4}=\sum_{1}^{Q}\left(\frac{\left(a-a_{1}\right)^{2}}{\beta_{b}^{2} b_{j}^{4}}+\frac{\left(b-b_{1}\right)^{2}}{\beta_{b}^{2} b_{j}^{2}}+\frac{\left(b-b_{1}\right)^{2}\left(c-c_{1}\right)^{2}}{\beta_{b}^{2} b_{j}^{4} b_{j}^{2}}\right), \\
& w_{5}=\sum_{1}^{Q}\left(\frac{\left(b-b_{1}\right)^{2}\left(c-c_{1}\right)^{2}}{b_{j}^{2}}\left(\frac{1}{\beta_{b}^{2}}-\frac{1}{\beta_{b}^{2} b_{j}^{2}}\right)\right) .
\end{aligned}
$$

Among, $b=\sqrt{\left(a-a_{1}\right)^{2}+\left(b-b_{1}\right)^{2}}$

According to the above formula, the coordinates can be obtained as:

$$
\begin{aligned}
& b_{1}=\frac{1}{\operatorname{det}(w)}\left(w_{4} w_{5}-w_{3}^{2}\right), \\
& b_{2}=\frac{1}{\operatorname{det}(w)}\left(w_{1} w_{5}-w_{2}^{2}\right), \\
& b_{3}=\frac{1}{\operatorname{det}(w)}\left(w_{1} w_{4}-w_{2}^{2}\right) .
\end{aligned}
$$

According to the calculation, the calculation of the geometric distance between the target objects has a great relationship with the angle between the objects.

2.3. Computer-Assisted Teaching. Teaching by computer is a service that uses computers to replace or help instructors to complete their tasks and impart knowledge. Intelligent computer teaching assistance system refers to the use of computers to help and replace teachers to perform part of the teaching tasks, transfer teaching information, impart knowledge and training skills to students, and serve students directly. This paper evaluates the teaching effect of the intelligent computer teaching assistance system, and thinks that the effect is better than the traditional teaching. The study of the computer-assisted teaching model, also known as the information-based instruction model, requires an epistemological and values-based approach, and analyze from different dimensions. The specific situation is shown in Figure 6:

In the process of traditional physical education, teachers mainly give oral explanations and action simulations to sports items, but the help to students is limited. The defects of teaching are conducive to improving the effect of physical education.

In traditional teaching methods, teachers usually use language and text to teach knowledge points to students. For ensuring the intuitive effect, teachers will use models and illustrations to illustrate the classroom content. However, not all teaching content can find suitable reference objects. For example, the thermal motion of molecules cannot be displayed with physical images. Computer-assisted teaching can make up for the shortcomings of traditional teaching methods with the help of pictures and texts, make the content that is difficult to tell in traditional teaching more intuitive, concentrate students' attention, deepen the impression and understanding, and improve teaching efficiency. Computer-assisted teaching has not only changed the teaching methods, but also promoted the reform of teaching concepts. In the era of knowledge explosion, how to find the required information among all kinds of complicated information is the key. Computer-assisted teaching can cultivate students' imagination, diverge students' thinking, improve students' problem-solving ability, and change the result-oriented concept in the traditional sense, pay 


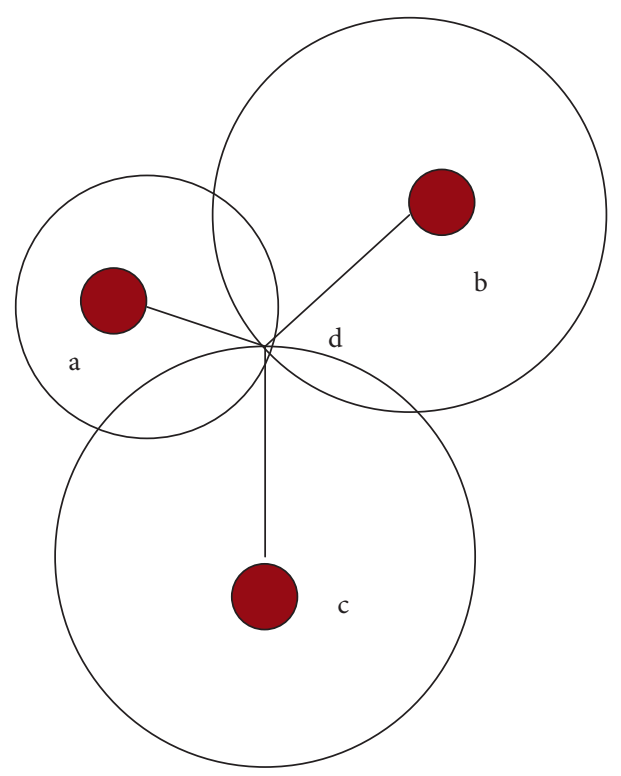

Figure 3: Coordinate axis measurement.

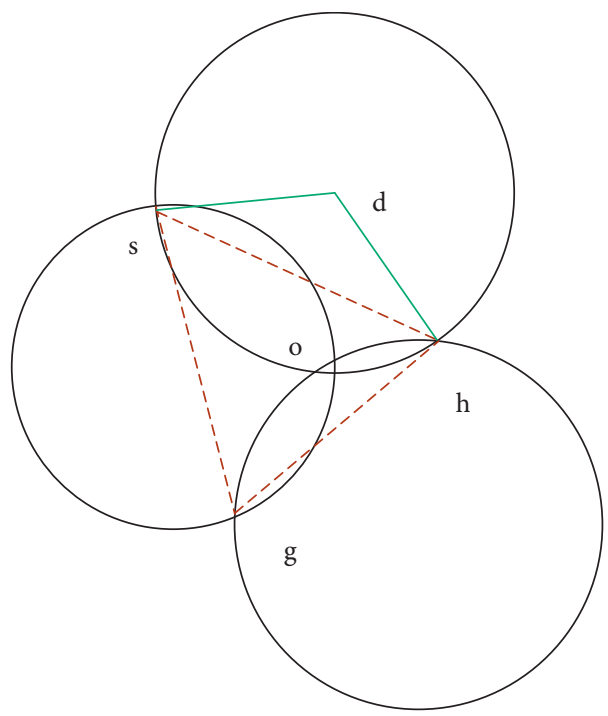

FIgURE 4: Triangulation positioning.

attention to the process of problem-solving and learn methods so that it can control different types of knowledge and promote own ability. In the traditional teaching model, we take the teacher as the center of the classroom, thinking that as long as the teacher describes the knowledge, the teaching results show that this teaching method is not conducive to students' subjective initiative, and is not conducive to students' understanding of knowledge. Introducing computer-assisted teaching into the classroom, transforming the traditional teacher-centered model to student-centered. In the teaching process, Increased focus on students' understanding of knowledge, and help students understand the nature and laws of things reflected in the current learning content and the internal relations between the things and other things, so as to achieve a true grasp of knowledge. The current computer teaching aid system has achieved many results. The introduction of software engineering methods into the development of computer-aided teaching systems has promoted the engineering of curriculum design, such as using artificial intelligence technology to simulate the behavior of "tutors" and improve student performance.

Computer technology and teaching theory are two important factors that affect computer-assisted teaching. Since the middle of the last century, computer technology has undergone a series of developments, and computer operations have become more convenient and smaller in size, there are more and more high-level languages, the running speed is getting faster and faster, and the computer storage is getting bigger and bigger. The combination of computer and communication technology is used 


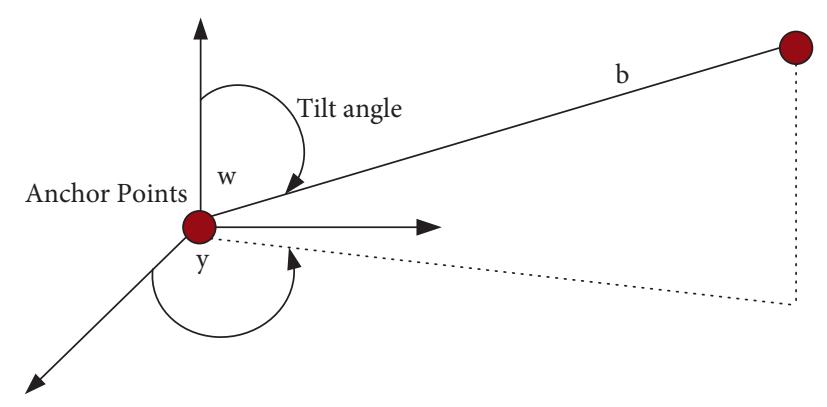

Figure 5: Angle and distance.

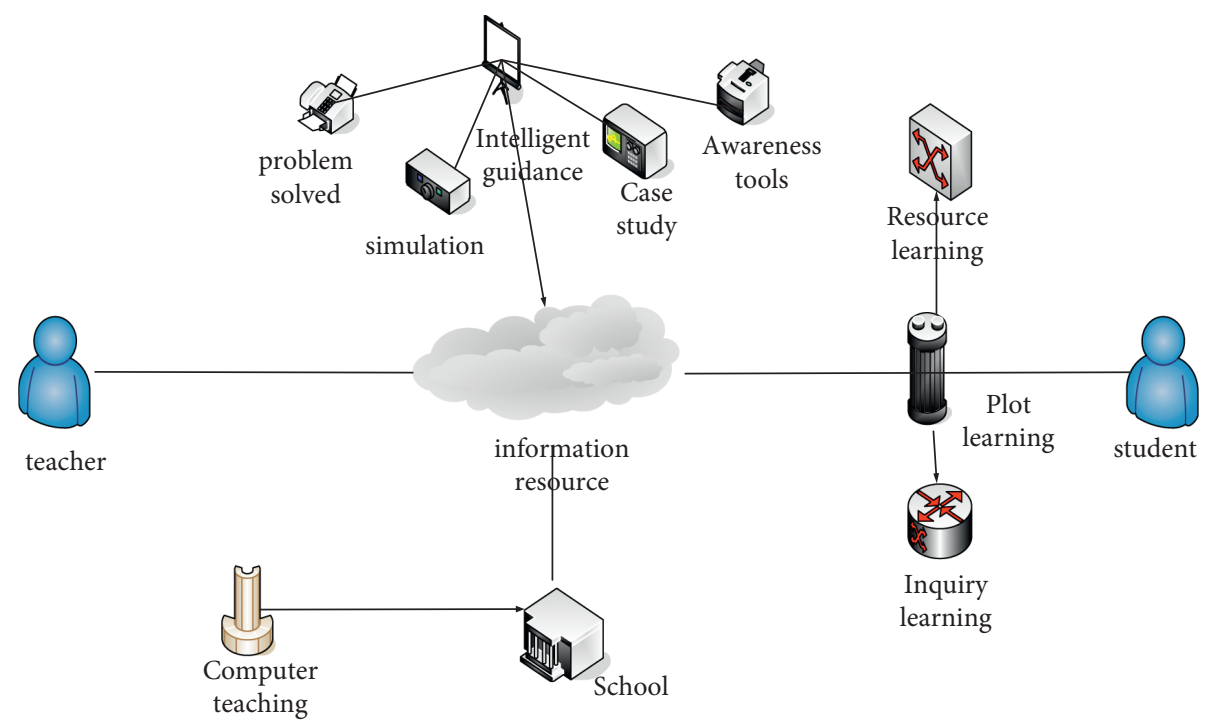

Figure 6: The structure of computer-assisted teaching mode.

extensively in the sphere of productive society. providing technical support for processing educational information. The United States first developed a computer teaching aid system in the last century. The computer can generate exercises based on classroom needs. The real teaching aid system is "PLATO," which is connected to a large computer and can ask different questions about classroom knowledge. When students answer, they can touch the screen or buttons.

The combination of wireless sensor networks and the Internet of Things improves the efficiency of teachers guiding students' experiments. In college physics classroom experiments, students often encounter various problems. In traditional teaching methods, students often raise their hands to signal when they encounter problems, and then teachers go over to guide them. However, based on the current situation of college physics experiment teaching, it is not possible to provide one-toone guidance by teachers. In fact, the positioning of sensor nodes can be realized only by relying on the wireless sensor network itself, and the guidance function can be quickly realized online when combined with the Internet of Things technology.

\section{Realization Experiment of Wireless Sensor and Intelligent Computer-Assisted Teaching in Physical Education and Training}

3.1. Objects. Aiming at the use of the combination of wireless sensors and smart computers in physical education, we conducted a questionnaire survey on different types of schools. The specific results are as follows:

According to the data in Table 1, this experiment investigated the understanding of wireless sensors and smart computers among students of first tier universities, second tier universities, and third tier universities. The total number of people surveyed was 230, of which boys accounted for $68.2 \%$, girls accounted for $31.8 \%$, and the number of third tier universities students accounted for $32.7 \%$ of the total survey number, second tier universities accounted for $30 \%$ of the total number of surveyed students, and first tier universities accounted for $37.3 \%$ of the total surveyed people. The numbers of the three types of institutions are relatively even. 
TABLE 1: Basic situation of the survey.

\begin{tabular}{lccc}
\hline \multirow{2}{*}{ Basic situation } & Number of people & $\begin{array}{c}\text { Proportion } \\
(\%)\end{array}$ \\
\hline \multirow{2}{*}{ Gender } & Male & 157 & 68.2 \\
& Female & 73 & 31.8 \\
\hline \multirow{3}{*}{ School category } & Junior college & 75 & 32.7 \\
& Niben & 69 & 30 \\
& One & 86 & 37.3 \\
\hline
\end{tabular}

As teaching tools, wireless sensors and smart computers not only need to be used in schools, but more importantly, they need to be used proficiently. Both teachers and students need to be proficient, so that smart computers can play a role in physical education.

According to the data in Table 2, in this survey, we separately investigated the proficiency of teachers and students on smart computers. $70 \%$ of teachers can master the basic operations of smart computers, $8 \%$ of teachers can master the complex operations of smart computers, and $22 \%$ of teachers can master the operations of smart computer websites, the proportion of teachers who can master the offline operation of smart computers is $49 \%$, and the proportion of teachers who can master the online operation of smart computers is $55 \%$. Generally speaking, the level of mastery is relatively average. $70 \%$ of students can master the basic operations of smart computers, $25 \%$ of students can master the complex operations of smart computers, and $44 \%$ of students who can master smart computer website operations, the proportion of students who can master the offline operation of smart computers is $55 \%$, and the proportion of students who can master the online operation of smart computers is $41 \%$. From the perspective of students' mastery, the number of people who use complex operations is relatively small, and they are generally proficient in using smart computers.

3.2. Validity of Student Questionnaire. This article's mastery of intelligent computers in colleges and universities is basically derived from questionnaire surveys, to understand the use of intelligent computers in colleges and universities in sports classrooms from different angles. Therefore, the questions in the questionnaire need to be taken seriously, and some unnecessary questions should be deleted.

According to the data in Table 3, in the questionnaire survey of students, we analyzed the structure and proportion respectively, and scored them by 10 experts. In terms of structure, $40 \%$ of the experts think the structure is excellent, $40 \%$ of the experts think the structure is very good, and $20 \%$ of the experts think the structure is general. In terms of content, $550 \%$ of the professionals thought the survey instrument had good content and 50\% of the professionals thought the survey instrument had good substance. From the overall data, the quality of the student questionnaire survey is still good, which meets the needs of the survey.

3.3. Validity of Teacher Questionnaire. According to the data in Table 4, like the questionnaire validity survey for students, we also analyze the validity of the teacher questionnaire from two aspects: structure and content. First of all, in terms of structure, $50 \%$ of the experts think the structure is excellent, $40 \%$ of the experts think the structure is very good, and $10 \%$ of the experts think the structure is general. In terms of content, $30 \%$ of the professionals thought the survey instrument was very good and $40 \%$ thought the subject matter of the questionnaire was very good, and $30 \%$ of the experts think that the content is very general. So from the overall data, the quality of the teacher's questionnaire survey is average, does not deviate from the target, and meets the needs of the survey.

\section{Realization of Wireless Sensor and Intelligent Computer-Assisted Teaching in Physical Education and Training}

4.1. Recognition of Intelligent Computer-Assisted Teaching. Although the combination of wireless sensors and smart computers can improve teaching efficiency, some teachers may not be able to accept it because the teaching methods are too novel, or even students cannot adapt to the classroom.This experiment is in the scope of the normal physical education classroom, and there is no obvious difference from the traditional classroom. According to the results of intelligent teaching, the classroom effect of this experiment is better and the degree of student participation is higher.

According to the data in Figure 7, most students support the use of intelligent computer technology in physical education classrooms. Supporting students believe that physical education is a practical subject, to get a better grasp of it. The intelligent aided learning and teaching computer system can provide converted images for the classroom, which significantly increases the productivity of the physical education classroom. According to the data, $40 \%$ of the pupils were very lectures pleased with the Smart Computer System in Physical Education class and 67\% were pleased with the usage of the Smart Computer System in Physical Education class, and $22 \%$ of students disagree with the use of intelligent computer technology in physical education classes.

From the perspective of smart computer classroom content, $20 \%$ of students are very satisfied with the classroom content, $27 \%$ think the classroom content is good, and $35 \%$ are dissatisfied with the physical education class. Dissatisfied students think that the courseware is too single to highlight the main points.

With the popularization of intelligent computer technology, faculties and schools are increasingly focusing on the use of intelligent computers. According to the data in Figure 8 , only $14.2 \%$ of teachers who approved the introduction of intelligent computer systems in classrooms often use them in physical education classrooms, $30 \%$ of teachers occasionally use it in physical education classes, and $12 \%$ of teachers never use it in physical education classes. Among the proportion of teachers who do not approve the introduction of intelligent computer systems into classrooms, no teacher often uses computer-assisted teaching in physical education classes, and $7 \%$ of teachers occasionally use 
TABLE 2: Intelligent computer proficiency survey.

\begin{tabular}{llccccc}
\hline & Category & Basic operation & Complex operation & Website operation & Offline operation & Online operation \\
\hline \multirow{2}{*}{ Teacher } & Number of people & 70 & 8 & 22 & 49 & 55 \\
& Proportion (\%) & 70 & 8 & 22 & 49 & 55 \\
\hline \multirow{2}{*}{ Student } & Number of people & 161 & 57 & 102 & 126 & 54 \\
& Proportion (\%) & 70 & 25 & 44 & 41 & 55 \\
\hline
\end{tabular}

TABLE 3: Validity of student questionnaire.

\begin{tabular}{lcccc}
\hline Validity & Excellent & Very good & General & Ineffective \\
\hline Structure & 4 & 4 & 2 & 0 \\
Proportion (\%) & 40 & 40 & 20 & 0 \\
Content & 5 & 5 & 0 & 0 \\
Proportion (\%) & 50 & 50 & 0 & 0 \\
\hline
\end{tabular}

TABLE 4: Validity of teacher questionnaire.

\begin{tabular}{lcccc}
\hline Validity & Excellent & Very good & General & Ineffective \\
\hline Structure & 5 & 4 & 1 & 0 \\
Proportion (\%) & 50 & 40 & 10 & 0 \\
Content & 3 & 4 & 3 & 0 \\
Proportion (\%) & 30 & 40 & 30 & 0 \\
\hline
\end{tabular}
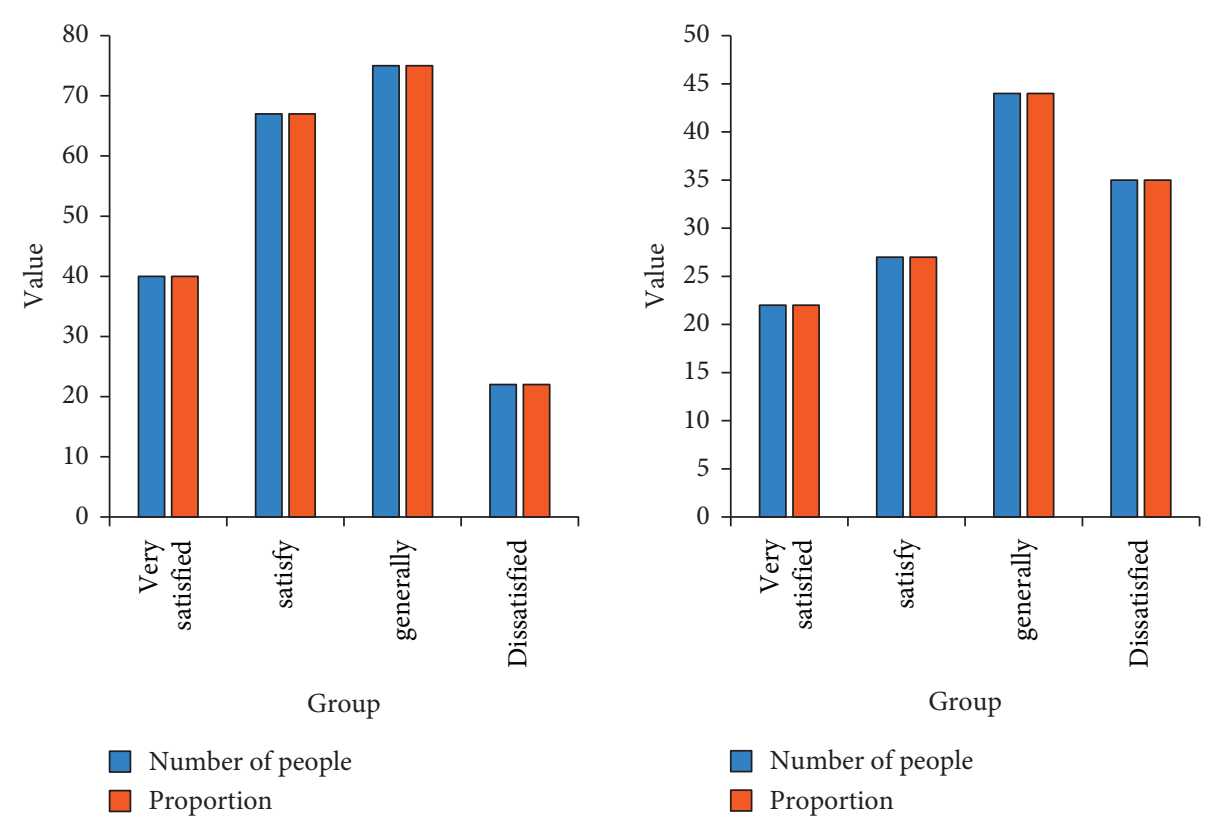

Figure 7: Analysis of recognition of intelligent computer-assisted teaching.

computer-assisted teaching in physical education classes, another $47 \%$ of teachers have never used computer-assisted teaching in physical education classrooms. It can be seen from these data that most teachers still recognize the computer-assisted teaching system, but the frequency of use is still not enough. This may be linked to the level of proficiency in the operation. Faculties and schools can organise relevant training.

4.2. The Effect of Computer-Assisted Teaching. This experiment investigates and analyzes the use of intelligent computer-assisted teaching systems in physical education classrooms and the teaching effects of computer-assisted teaching. The specific data are as follows:

According to the data in Figure 9, 46\% of students who use computer-assisted teaching in physical education classrooms think that the classroom effect is very good, and they are very impressed; $55 \%$ of students think that the classroom effect is very general, and it is no different from normal classroom teaching, $18 \%$ of students believe that the classroom effect is very poor, and the lecture speed is too fast for in-depth understanding. For the problems encountered in the classroom, $46 \%$ of students tend to take good notes 

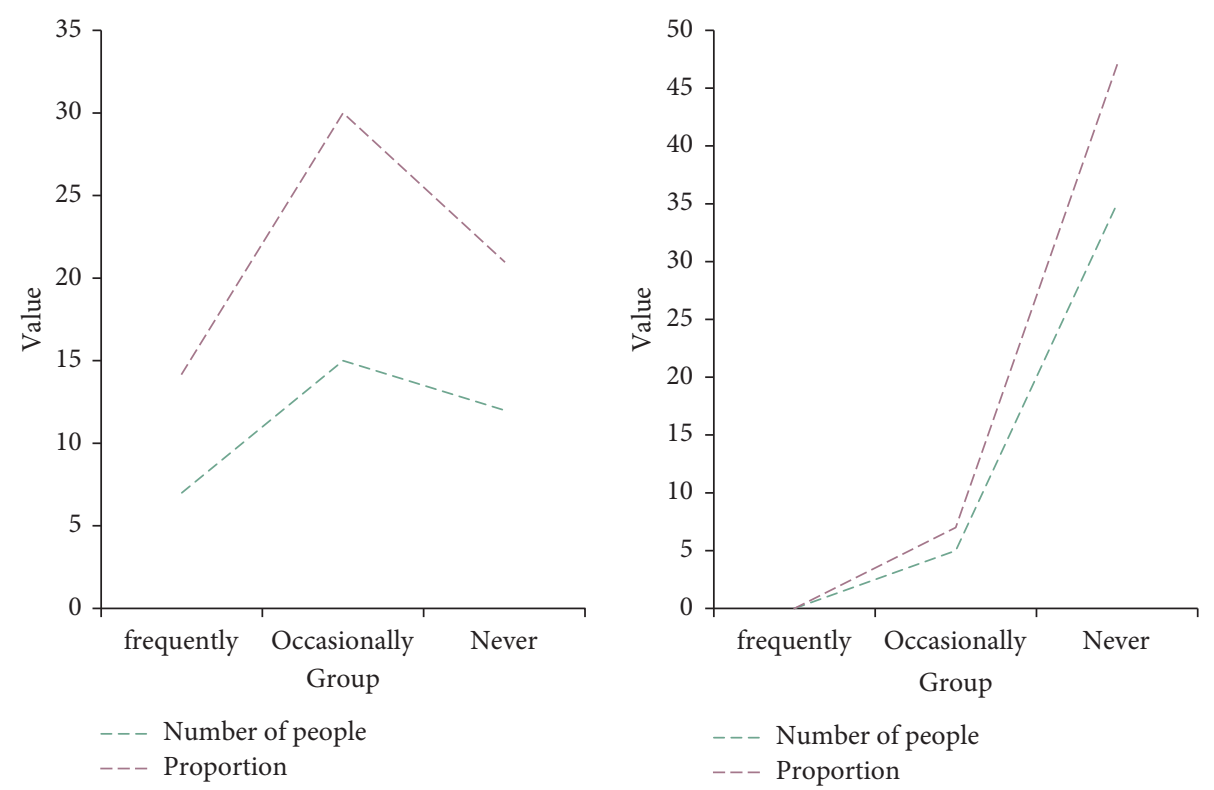

Figure 8: Analysis of teacher recognition.
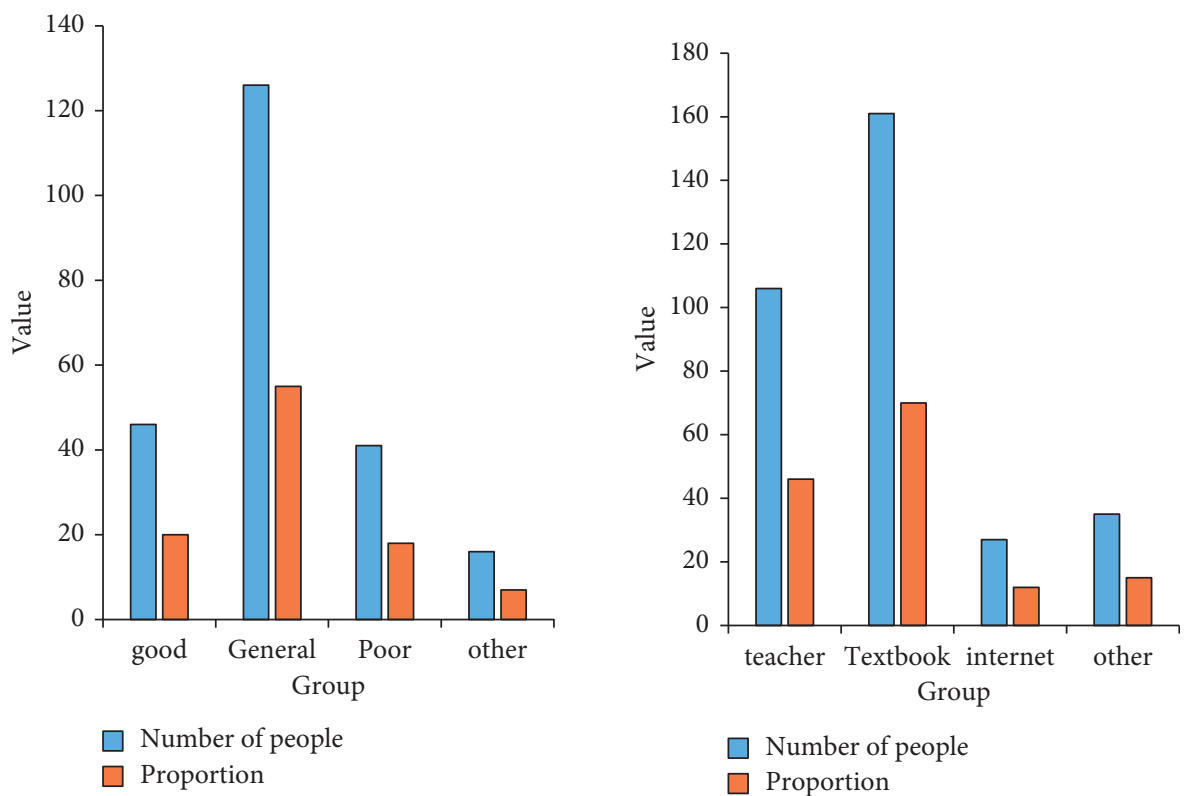

Figure 9: The effect of computer-assisted teaching.

and ask the teacher for advice after class; $70 \%$ of students tend to consult textbooks to solve them on their own; $12 \%$ of students tend to solve them through a computer network. From this data, it is evident that in order to solve issues in the PE classroom, students have low awareness of the Internet and low use of computers.

4.3. Effect Analysis. According to the data in Figure 10, only $10 \%$ of students often use smart computers outside the classroom, $47 \%$ of students occasionally use computerassisted learning, and $40 \%$ of students rarely use computer- assisted teaching, another $3 \%$ of students never use computer-assisted learning. Among the students who use intelligent computer-assisted learning, $10 \%$ of the students think the effect is very good, $50 \%$ of the students think the effect is very general, $20 \%$ of students think it does not help. From the overall data, most students will use computers for physical education, but the learning effect is not good.

Computer-aided teaching is based on cognition and applies artificial intelligence technology to computer-aided teaching. Intelligent CAI (ICAI) separates teaching content and teaching strategy, and dynamically generates content and teaching strategy suitable for individualized teaching 

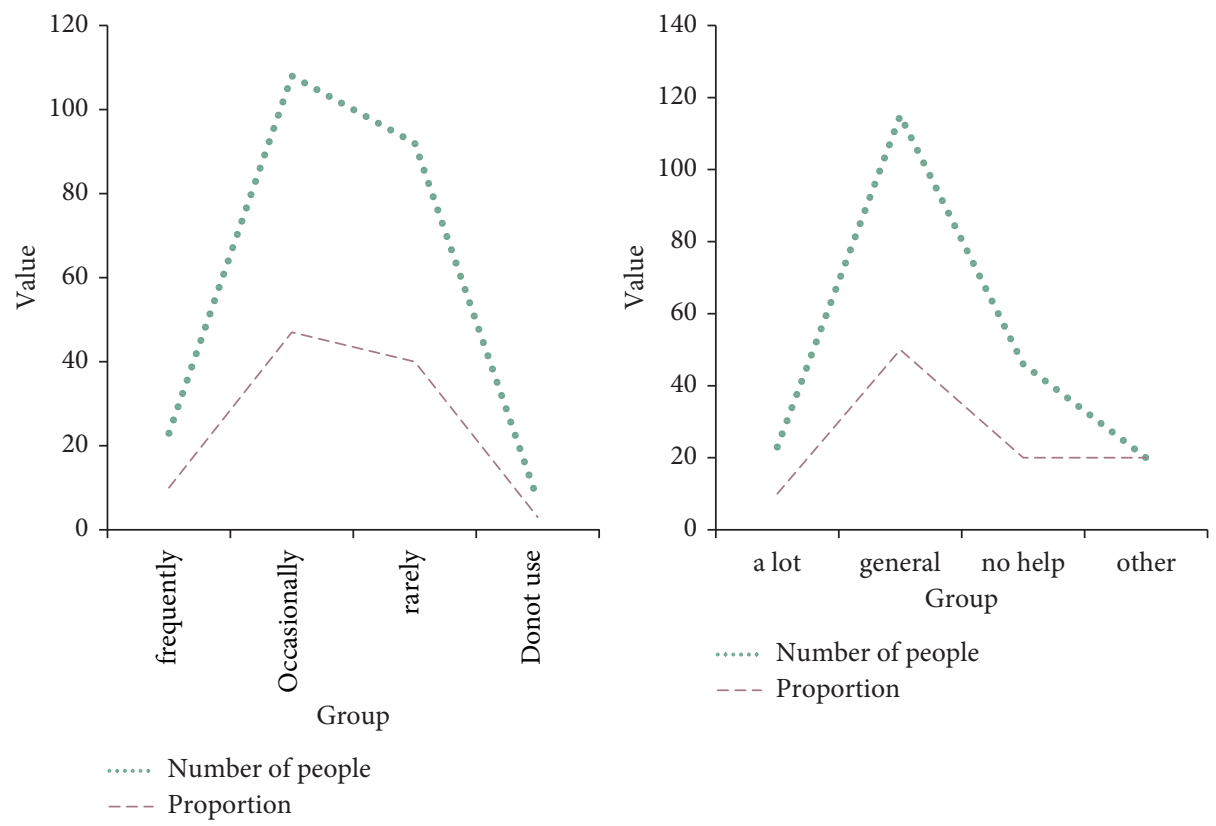

Figure 10: Analysis of learning effect.

through search and judgment of intelligent system combined with wireless sensor network technology according to the detailed information provided by students' cognitive model. . Through the intelligent diagnosis mechanism, it can judge the learning level of students, analyze the reasons for students' mistakes, and at the same time, propose changes to students and suggestions for further learning content.

\section{Conclusions}

As the strategy of "developing the country through science and education" progresses, society is paying more and more attention to education. How to improve the efficiency of Sports training to develop more people for society has become the current pursuit goal. Since the advent of computers, computers have developed at an astonishing speed, their performance has continued to increase, and their prices have gradually fallen. This has provided technical and material support for the creation and development of computer-assisted teaching systems. This article aims to explore the role of wireless sensors and intelligent computer-assisted teaching in physical education and training. In this article, the following tasks are mainly completed: (1) Analysis of the current state of the art in radio frequency sensor research, and its concept, structure, characteristics, application fields and other aspects are explored. (2) Introduced the research progress of wireless sensors, It also discusses the structure of radio signal networks and the prospects of their adoption in the field of sports.

\section{Data Availability}

Data sharing is not applicable to this article as no new data were created or analyzed in this study.

\section{Conflicts of Interest}

The author states that this article has no conflict of interest.

\section{Acknowledgments}

The author(s) received no financial support for the research, authorship, and/or publication of this article.

\section{References}

[1] M. Xu, Y. Zhai, Y. Guo et al., "Personalized training through Kinect-based games for physical education," Journal of Visual Communication and Image Representation, vol. 62, pp. 394401, 2019.

[2] I. Khan, F. Belqasmi, R. Glitho, N. Crespi, M. Morrow, and P. Polakos, "Wireless sensor network virtualization: early architecture and research perspectives," Network IEEE, vol. 29, no. 3, pp. 104-112, 2016.

[3] R. Amin and G. P. Biswas, "A secure light weight scheme for user authentication and key agreement in multi-gateway based wireless sensor networks," Ad Hoc Networks, vol. 36, pp. 58-80, 2016.

[4] T. Kunz and B. Tatham, "Localization in wireless sensor networks and anchor placement," Journal of Sensor \& Actuator Networks, vol. 1, no. 1, pp. 36-58, 2018.

[5] Z. Liu, Z. Li, M. Li, W. Xing, and D. Lu, "Path reconstruction in dynamic wireless sensor networks using compressive sensing," IEEE/ACM Transactions on Networking, vol. 24, no. 4, pp. 1948-1960, 2016.

[6] J. Long, M. Dong, K. Ota, and A. Liu, "Achieving source location privacy and network lifetime maximization through tree-based diversionary routing in wireless sensor networks," IEEE Access, vol. 2, no. 2, pp. 633-651, 2017.

[7] S. H. Seo, J. Won, S. Sultana, and E. Bertino, "Effective key management in dynamic wireless sensor networks," IEEE Transactions on Information Forensics and Security, vol. 10, no. 2, pp. 371-383, 2017. 
[8] S. D. de Carvalho, F. R. de Melo, E. L. Flôres, S. R. Pires, and L. F. B. Loja, "Intelligent tutoring system using expert knowledge and Kohonen maps with automated training," Neural Computing and Applications, vol. 32, no. 17, pp. 13577-13589, 2020.

[9] S. Lee, H. Noh, J. Lee, K. Lee, and G. G. Lee, "Foreign language tutoring in oral conversations using spoken dialog systems," IEICE - Transactions on Info and Systems, vol. 95, no. 5, pp. 1216-1228, 2017.

[10] S. Rum and M. A. Ismail, "Metacognitive support accelerates computer assisted learning for novice programmers," $E d u$ cational Technology \& Society, vol. 20, no. 3, pp. 170-181, 2017.

[11] A. Lounis, A. Hadjidj, A. Bouabdallah, and Y. Challal, "Healing on the cloud: secure cloud architecture for medical wireless sensor networks," Future Generation Computer Systems, vol. 55, pp. 266-277, 2016.

[12] T. Zheng, M. Gidlund, and J. Akerberg, "WirArb: a new mac protocol for time critical industrial wireless sensor network applications," IEEE Sensors Journal, vol. 16, no. 7, pp. 2127-2139, 2016.

[13] A. Remah and E. Khaled, "Performance and challenges of service-oriented architecture for wireless sensor networks," Sensors, vol. 2017, no. 17 536, pp. 536-575, 2017.

[14] S. Vercruysse, "Effectively training physical education teachers to implement injury preventive strategies into their pe lessons," British Journal of Sports Medicine, vol. 51, no. 4, pp. 412-404, 2017.

[15] M. Couto, J. Marques, D. Silva, M. Paiva, T. Jacinto, and R. Câmara, "What physical education teachers know about asthma: impact of a training course," Journal of Investigational Allergology and Clinical Immunology, vol. 29, no. 5, pp. 392-394, 2019.

[16] P. Boselin, V. Sakkthi, A. Babu, T. C. Anand, and S. Sophia, "Mobility assisted dynamic routing for mobile wireless sensor networks," Social Science Electronic Publishing, vol. 3, no. 1, pp. 9-19, 2017.

[17] W. Sun and Y. Gao, "The design of university physical education management framework based on edge computing and data analysis," Wireless Communications and Mobile Computing, vol. 20218 pages, 2021.

[18] M. Udo and T. Katayama, "A study on the in-service training programs for physical educators of junior and senior high schools in Japan," Research of Physical Education, vol. 13, no. 4, pp. 305-311, 2016.

[19] J. Li, "Research on the reform and innovation of preschool education informatization under the background of wireless communication and virtual reality," Wireless Communications and Mobile Computing, vol. 2021, pp. 3176309:13176309:6, Article ID 3176309, 2021.

[20] H.-S. Lee and Jeong-Ae, "Exploring direction and system of analyzing physical education textbooks to," Korean Education Inquiry, vol. 34, no. 1, pp. 115-133, 2016. 